\title{
Simulating Shot Noise in Electron Holograms -- Recorded Digitally
}

\author{
E. Voelkl
}

\section{HoloWerk LLC, 3517 Grimes Ranch Rd, Austin, TX 78732}

Although field-emission electron-sources for modern STEM/TEMs are considered highly coherent compared to $\mathrm{LaB}_{6}$ or Tungsten emitters, they provide a limited coherence compared to e.g., a laser in light optics. This is due to the facts that electrons are Fermions (i.e., particles with $1 / 2$ integer spin) and therefore adhere to the Pauli-exclusion-principle keeping them from occupying the same quantum number (identical energy level and spin), whereas photons are characterized as Bosons (with an integer spin) and thus have no qualms of being in the same quantum state.

Specifically the lateral coherence relating directly to the effective source size as viewed from the final image plane (also impacted by stray fields from the environment) is directly affecting the fringe contrast of the hologram and thus the signal-noise level in the reconstructed phase and amplitude images. The generally accepted practice of adjusting the illumination to provide a very small convergence angle, see chapter 4 in [1], has the serious disadvantage that the electron dose on the image recording device, usually a CCD camera, is rather small, around 100-500. Thermal drift instabilities, specifically of the biprism and the illumination system, put a limit to the useful exposure time for the image recording device, usually around 1-5s.

Thus, every electron counts --- and the question arises, how effective an image recording device like a modern CCD camera is for off-axis type holography. This topic has been discussed in chapters 5 and 9 of [1]. Another way to address this question is to estimate the signal noise levels in the amplitude and phase of holograms based on a single electron build-up of the hologram. The intensity distribution $I=I(m, n)$ for an off-axis type hologram is given in its simplest form as:

$$
I=P\left[A^{2}+B^{2}+2 \mu A B \cos \left(2 \pi \boldsymbol{r} \boldsymbol{s}+\Delta \varphi+\varphi_{0}\right)\right],
$$

where $A=A(m, n)$ is the amplitude of the target wave, $B=B(m, n)$ is the amplitude of the reference wave, $\mu$ is the coherence factor (or fringe contrast as measured for $A=B$ and no target and $0 \leq \mu<1$ and includes the CTF of the camera), $\Delta \varphi=\Delta \varphi(m, n)$ is the phase difference between the target and reference wave, $\varphi_{0}$ is a constant phase factor describing e.g., the position of the interference fringes with respect to the CCD camera, $m$ and $n$ the pixel positions on the $\mathrm{CCD}$ (with $0 \leq m \leq M$ and $0 \leq n \leq N$ ) with $M N$ pixels, $\boldsymbol{r}=(m, n)$ the position of a pixel on the CCD camera, $\boldsymbol{s}$ the sampling rate for the interference fringes and $P$ the average number of electrons over $M N$ pixels, ignoring the effects of finite pixel size, dark current and gain variations.

For simulating the statistical behavior of electrons building up the final hologram, a program was written using the DigitalMicrograph ${ }^{\circledR}$ scripting language and their Random() operator providing values with a statistical distribution corresponding to white noise. In Fig. 1, the simulated build-up of a hologram in several stages is displayed for the parameters $A=B=1$ and a sampling rate of (11, 18). The appearance of the simulated holograms is quite comparable to the actual recording of such a hologram build-up as discussed in [2-4]. 
The method was then used to predict the noise level in an actual electron hologram recorded with a Gatan CCD camera. For a sampling rate of $(5.12,12.8)$ and an average pixel value of 181 , which matched the actual conditions for a sample hologram, the standard deviation for the phase image was computed both for the simulated and experimental data. The result is displayed in Fig. 2 and the simulated and experimental phase noise agree within reason. This shot-noise-based approach to determining amplitude and phase noise in off-axis type holograms will be used to review the performance of CCD cameras for off-axis type holography applications.

\section{References}

[1] Introduction to Electron Holography, eds E Voelkl, L F Allard, D C Joy, Kluwer Academic / Plenum Publishers 1999.

[2] http://physicsweb.org/articles/world/15/9/1/1

[3] P G Merli, G F Missiroli and G Pozzi 1976 Am. J. Phys. 44 306-7

[4] A Tonomura, J Endo, T Matsuda, T Kawasaki and H Ezawa 1989 Am. J. Phys. 57 117-120
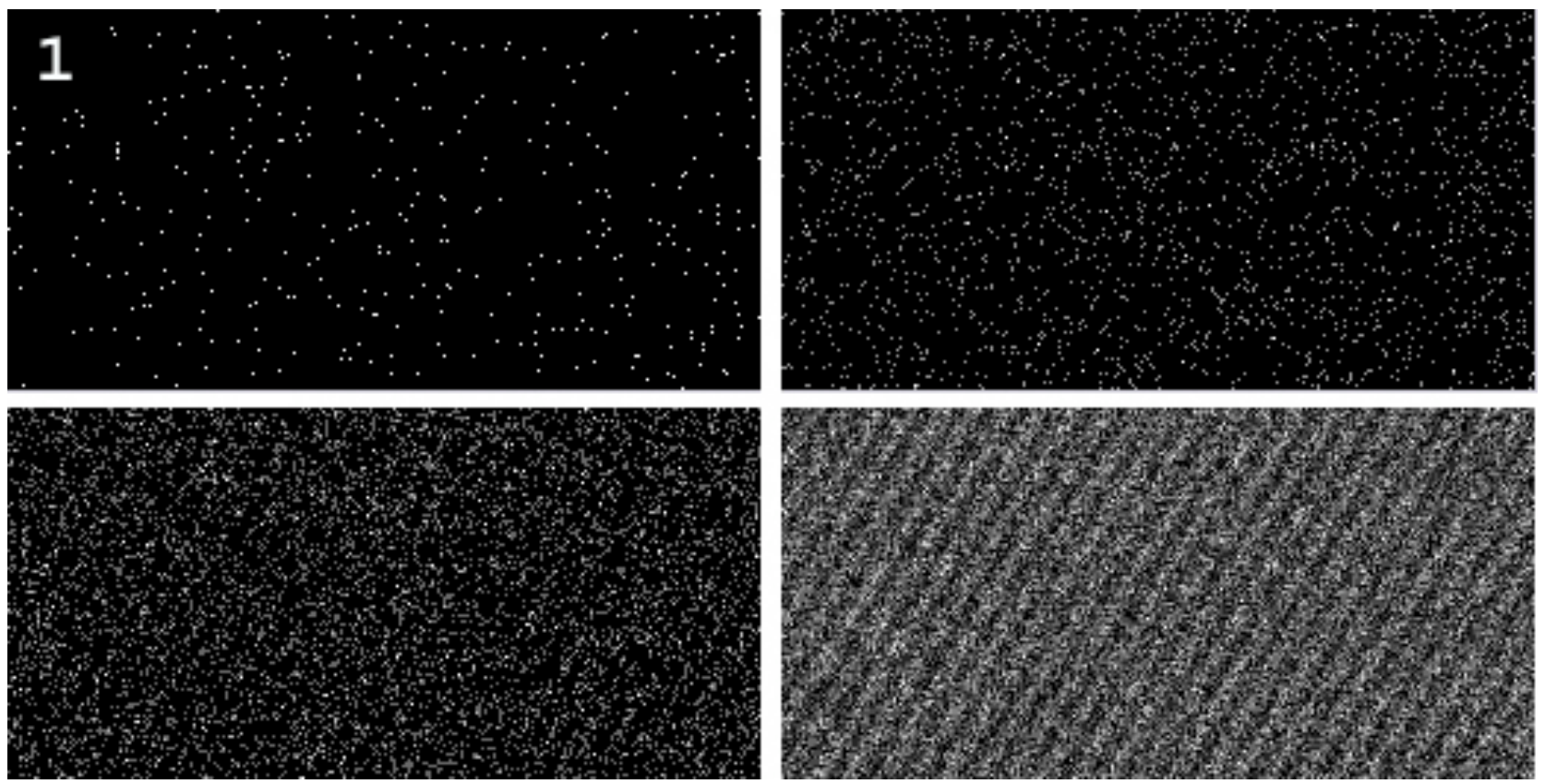

FIG. 1. Simulated build-up of an electron hologram for 400, 1900, 7.5k and 75k electrons, which compares to an average of $0.01,0.06,0.23$ and 2.3 electrons per pixel on a CCD camera.

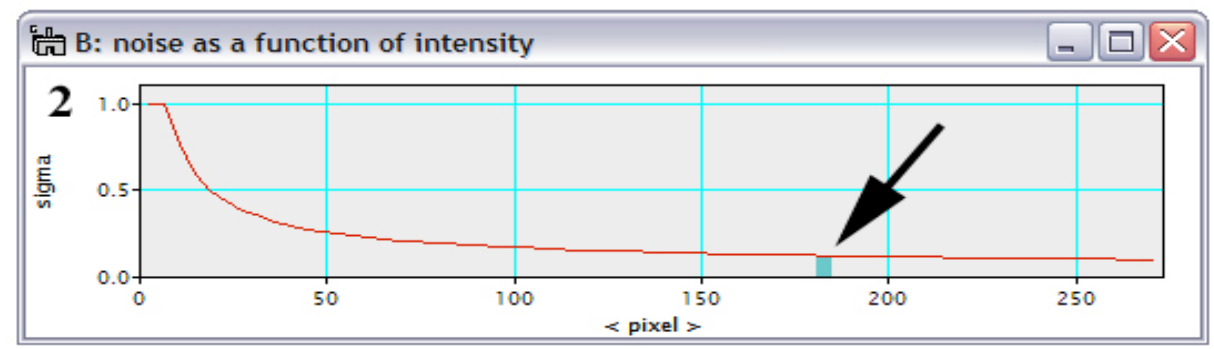

FIG. 2. Standard deviation of phase noise as a function of intensity on CCD camera. Experimental (see arrow) and simulated values (red) agree, although experimental value is lower than expected. 